COLORECTAL CANCER

\title{
Histological categorisation of fibrotic cancer stroma in advanced rectal cancer
}

\author{
H Ueno, A M Jones, K H Wilkinson, J R Jass, I C Talbot
}

See end of article for authors' affiliations

......................

Correspondence to:

Dr $\mathrm{H}$ Ueno, Department of

Surgery I, National

Defence Medical College,

3-2, Namiki, Tokorozawa

Saitama 359-8513

Japan;

veno@me.ndmc.ac.jp

Accepted for publication

15 October 2003
Background and aims: Based on conflicting reports regarding the role of the fibrotic stromal response in cancer development-namely, that a desmoplastic reaction can favour either the host or the tumour-it is clear that the role of the stromal response is varied. We have classified the fibrotic stroma of rectal adenocarcinoma penetrating the muscularis propria, based on histologically identified stromal components.

Methods: Three categories of stroma were used: mature-when the stroma was composed of mature collagen fibres (fine and elongated fibres into multiple layers); intermediate-when keloid-like collagen was intermingled with mature fibres; and immature-consisting of a myxoid stroma in which no mature fibres were included.

Results: In a data set of 862 patients, $53 \%$ of patients had mature fibrotic cancer stroma, 33\% had intermediate stroma, and $15 \%$ had immature stroma. Five year survival rates decreased as follows: mature stroma $(80 \%)$, intermediate stroma (55\%), and immature stroma (27\%). The adverse tumour phenotype, tumour cell budding (conspicuous isolated cells or small clusters of cancer cells), was observed in the cancer fronts in tumours with unfavourable fibrotic stroma $(p<0.0001)$. Based on multivariate analysis, categorised fibrotic stroma was selected as an independent prognostic parameter (hazard ratio 1.39; $95 \%$ confidence interval 1.17-1.64) together with tumour differentiation. By immunohistochemical examination, as maturation of the fibrotic stroma decreased, stromal T cells became significantly sparser. Furthermore, myofibroblasts were distributed extensively in immature fibrotic stroma compared with mature and intermediate fibrotic stroma.

Conclusion: The morphological categorisation of fibrotic cancer stroma highlights the role of the stromal response in relation to the behaviour and host immune reactions of rectal adenocarcinoma and would be a useful tool for predicting patient prognostic outcome. l: has been argued that fibrotic stroma could represent an attempt by the host to ward off tumour cells, thereby exerting antagonistic biological forces. Alternatively, this process may benefit the tumour, by neovascularisation and impeding access to host lymphocytes, macrophages, and other immune regulator cells. ${ }^{12}$ It has been demonstrated that L-3,4-dehydroproline induced inhibition of the desmoplastic response leads to an increase in spontaneous metastasis from experimental murine melanomas. ${ }^{2}$ Furthermore, the ability of tumour cells to induce a stromal response in the host tissue was observed to be inversely correlated with their metastatic potential in cloned Lewis lung carcinoma cells. ${ }^{3}$

These reports support the hypothesis that the desmoplastic response limits tumour aggressiveness and contradict the concept that tumours eliciting the greatest desmoplastic reaction behave more aggressively than those eliciting a less pronounced response. The latter was noted not only in colorectal cancer ${ }^{4}$ but also in other tumours, including breast carcinoma, ${ }^{5}$ pulmonary carcinoma, ${ }^{6}$ cholangiocarcinoma, ${ }^{7}$ and skin squamous carcinoma. ${ }^{8}$ This contradiction suggests that there are multiple processes in the desmoplastic reaction which have opposing effects on cancer behaviour. It is conceivable that such different processes may operate independently during neoplastic growth and could simultaneously occur at different locations in the same tumour. In order to understand such complex interactions between neoplastic cells and the surrounding matrix, it is necessary to examine not only the amount of fibrosis, which has hitherto been the pathological index for stromal reaction, but also its qualitative nature.
Fibrous connective tissue includes matrix components (for example, fibronectin, interstitial collagens, elastin, and glycosaminoglycans) and the cells responsible for matrix synthesis (for example, fibroblasts, myofibroblasts, and histiocytes). ${ }^{9}$ With due emphasis on these components, we have identified histological features and made a histological classification of fibrotic cancer stroma. ${ }^{10}$ The aim of the present study was to verify the clinicopathological significance of our classification, through study of the relationship of fibrotic cancer stroma to tumour behaviour, host cellular reactions, and survival outcome after surgery using a data set of patients with long term follow up.

\section{MATERIALS AND METHODS Patients}

Survival analyses were performed on a series of rectal cancer patients who underwent radical surgery at St Mark's Hospital between 1960 and 1980. The data set comprises 862 patients with rectal adenocarcinoma (226 in the lower rectum, 250 in the middle rectum, 317 in the upper rectum, and 69 at the rectosigmoid junction). This data set does not include patients with synchronous tumours or adenocarcinomas complicating familial adenomatous polyposis or inflammatory bowel disease. Patients who died within one month of surgery were also excluded. All tumours were confirmed pathologically as penetrating through the bowel wall to reach perirectal tissues. None of the patients received preoperative chemotherapy or radiation therapy. All patients were followed up until death or for at least five years; the average follow up period was 140 months. 


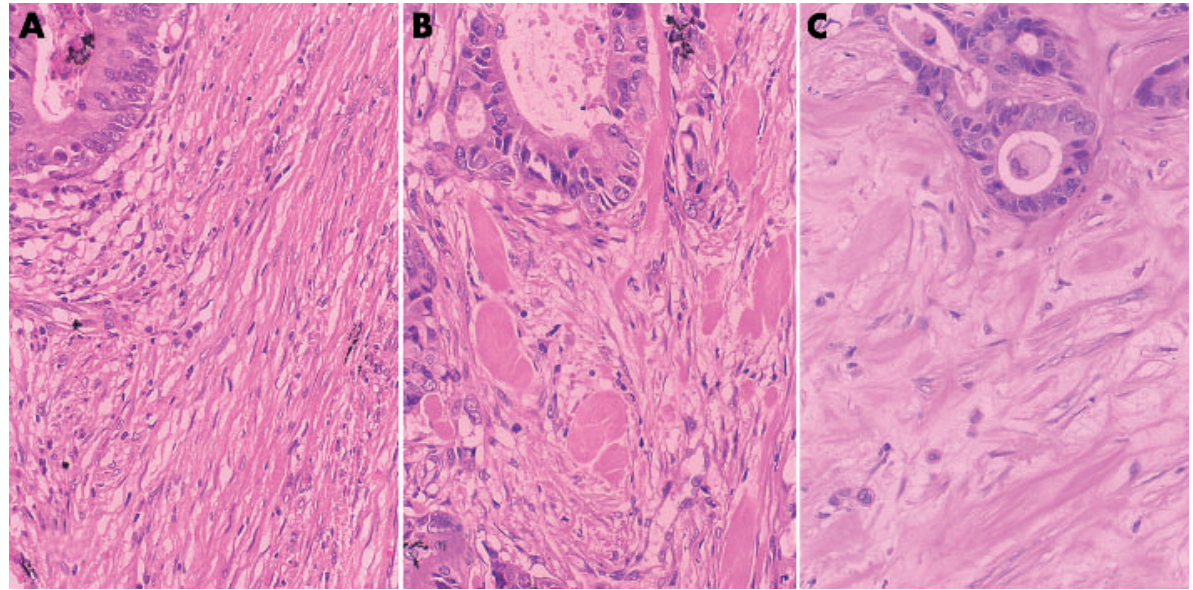

Figure 1 Maturation of fibrotic cancer stroma. (A) Mature stroma; (B) intermediate stroma; and (C) immature stroma. Magnification $\times 100$.

\section{Criteria for histological categorisation of fibrotic cancer stroma}

Fibrotic cancer stroma was classified as mature when composed of mature collagen fibres-that is, fine and elongated fibres with fibrocytes stratified into multiple layers-and intermediate when broad bands of collagen with brightly eosinophilic hyalinisation, similar to those seen in a keloid, ${ }^{10}$ were intermingled with mature collagen fibres (fig 1). Stroma was classified as immature fibrotic stroma when it consisted of randomly orientated keloid-like collagen bundles surrounded by myxoid stroma (fig 1). Stromal assessment in each case was made at the invasive frontal zone in the muscular layer or mesorectum, and was classified according to the most unfavourable stromal area (fig 2). The stroma around aggregations of acute inflammatory cells, which sometimes accompany gastrointestinal tumours and have an effect on the stroma, was excluded from consideration.

\section{Grading of tumour budding}

The morphological features of known prognostic importance such as tumour diameter, tumour type, differentiation, nodal involvement, and extramural venous invasion had previously been recorded in the pathological reports. In addition to these parameters, tumour budding at the cancer front was chosen as one pathological characteristic indicative of cancer behaviour. An isolated single carcinoma cell or a carcinoma cell cluster of fewer than five cancer cells, within the actively invasive region, was defined as a budding focus. ${ }^{11}{ }^{12}$ After meticulous investigation to choose a field where budding was most intensive, the number of budding foci was counted with a microscopic field of $700 \mu \mathrm{m}$ in diameter, as previously reported. ${ }^{11}$ Numbers of $0-4,5-9,10-19$, and $>20$ were scored as I, II, III, and IV, respectively.

\section{Immunohistochemistry}

Representative specimen blocks embedded in paraffin wax were serially sectioned at $5 \mu \mathrm{m}$ in cases selected randomly from each categorised group. One sectioned block of each of the specimens to be studied was stained with haematoxylineosin to confirm maturation of fibrotic stroma. Among 50 cases examined, 20 tumours had mature fibrotic stroma, 17 tumours had intermediate fibrotic stroma, and 13 tumours had immature fibrotic stroma. An immunostaining study was performed using the labelled streptavidin-biotin technique. Briefly, deparaffinised rehydrated sections were treated with $0.2 \%$ hydrogen peroxidase in methanol for three minutes to block endogenous peroxidase. For non-enzymatic epitope retrieval, sections were pressure cooked in a citrate buffer at pH 6.0 on full pressure for three minutes. Slides were allowed to cool and then sections were incubated in a 1:5 dilution of normal horse serum. Sections were incubated for one hour with mouse monoclonal antihuman $\mathrm{CD} 3$ (diluted 1:50 in phosphate buffered saline; Dako, Carpinteria, California, USA) or mouse monoclonal antihuman smooth muscle
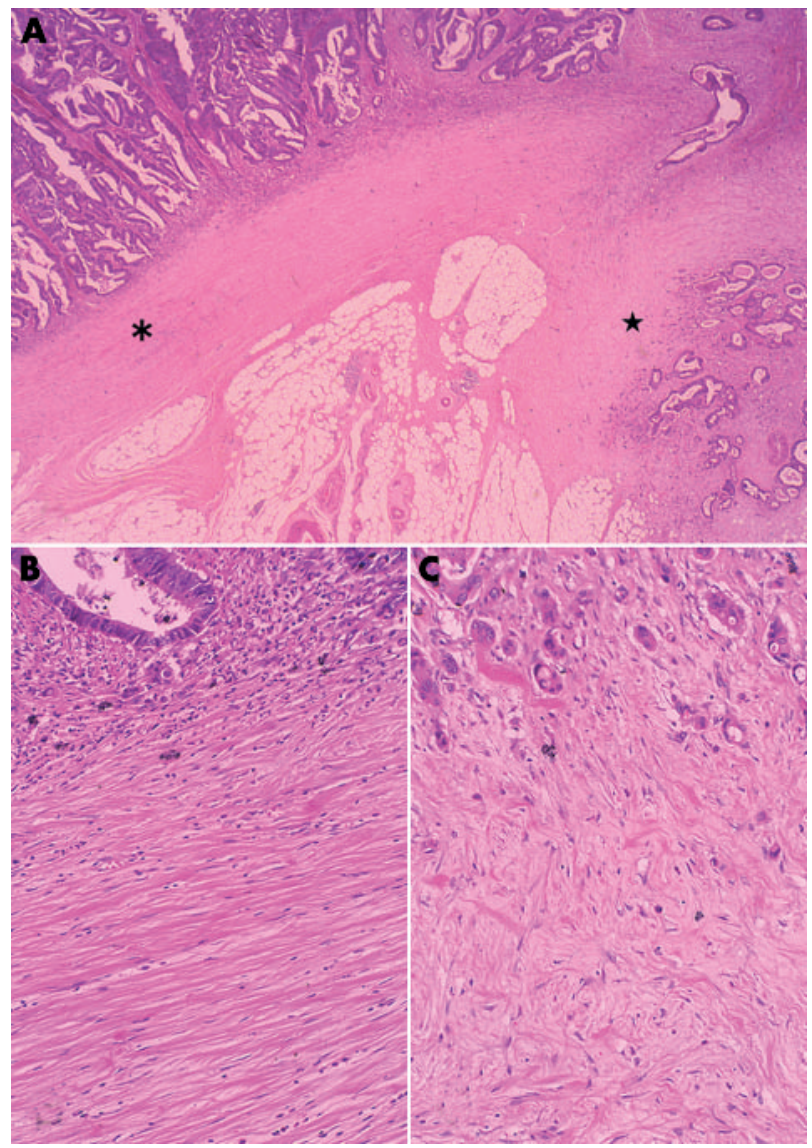

Figure 2 Heterogeneity of fibrotic cancer stroma. Mature fibrotic stroma shown in (B) (asterisk in (A)) and immature fibrotic stroma shown in (C) (star symbol in (A)) are observed in a tumour. (A) Invasive frontal area of a tumour. Assessment of maturation of fibrotic stroma was made based on the region of the most immature stroma. (A) $\times 2.7$; (B) $\times 50$; (C) $\times 50$. 


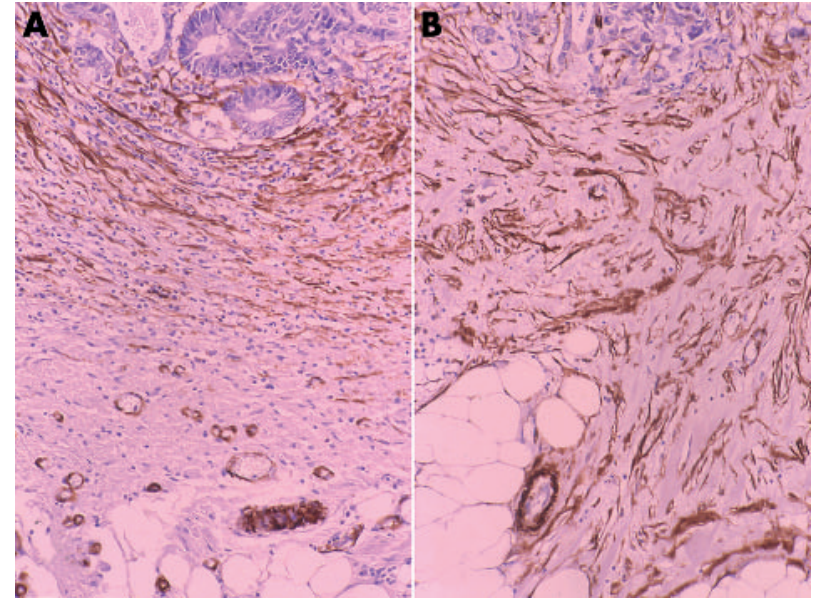

Figure 3 Localisation pattern of myofibroblasts in the reactive fibrous zone. (A) Confined: myofibroblasts become sparse with increased distance from the tumour $(\times 50)$. (B) Pervasive: myofibroblasts are observed densely, diffusely, in the whole reactive fibrous zone $(\times 50)$.

actin (diluted 1:500 in phosphate buffered saline; Dako) at room temperature. Biotinylated antimouse IgG (Vector, Burlingame, California, USA) (1:200 dilution) was used as the secondary antibody (30 minutes, room temperature). Following a wash in phosphate buffered saline, the avidinbiotin complex complex (Vector) was added for 30 minutes before the final DAB (Sigma-Aldrich, St Louis, Missouri, USA) was applied to develop the peroxidase.

\section{Distribution of T lymphocytes and myofibroblasts}

By looking at the immunohistochemistry for the pan $\mathrm{T}$ cell marker, CD3, we were able to assess the density of T cells in the invasive frontal region. The number of immunoreactive cells was counted within a microscopic field measuring $500 \mu \mathrm{m}$ in diameter. Three areas with the most abundant distribution were selected and the average number was calculated. With regard to the distribution of myofibroblasts identified by the antismooth muscle actin antibody, the localisation pattern in the reactive fibrous zone surrounding the cancer front was divided into two categories: A, confined; and B, pervasive (fig 3).

\section{Statistical analysis}

Survival rates were calculated using the Kaplan-Meier method, and comparisons were made by the log rank test. After the process of categorisation, each clinical and pathological variable was entered into a Cox's proportional hazard regression analysis to determine which parameter had an independent effect on postoperative survival. All variables except for cancer stroma and tumour budding had previously been recorded in the pathological reports. The unpaired $t$ test was used to check for a correlation between categories of

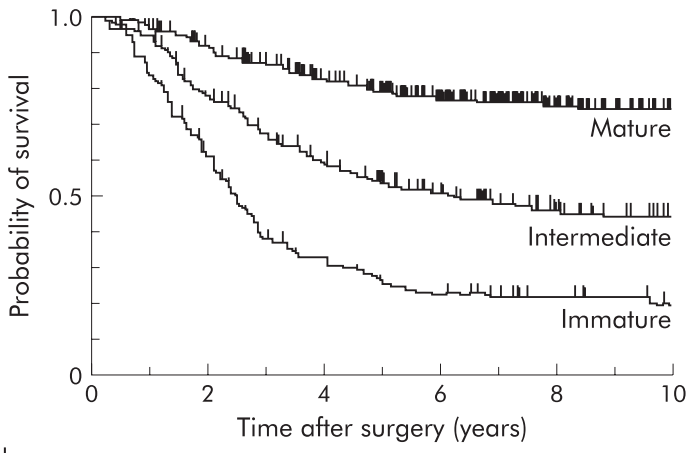

No at risk

$\begin{array}{lrrrrrr}\text { Mature } & 453 & 390 & 321 & 258 & 188 & 135 \\ \text { Intermediate } & 284 & 212 & 151 & 110 & 80 & 56 \\ \text { Immature } & 125 & 72 & 37 & 26 & 17 & 11\end{array}$

Figure 4 Kaplan-Meier estimates of cancer specific survival for 862 patients with rectal cancer penetrating the muscular layer, from the combined data sets according to the maturation of fibrotic cancer stroma (mature, intermediate, and immature). Note the correlation of patient survival and maturation of fibrotic cancer stroma. $p<0.0001$ between each group (log rank test).

fibrotic cancer stroma and the number of CD3 lymphocytes. The association between categorised fibrotic cancer stroma and tumour budding was analysed by Spearman's test. Maturation of fibrotic cancer stroma and distribution of myofibroblasts were submitted to Mann-Whitney's U test. Statistical calculations were performed using StatView ver.5.0 software (SAS Institute, Cary, North Carolina, USA).

\section{RESULTS \\ Impact of maturation of fibrotic cancer stroma on survival}

In the data set, $52.6 \%$ of patients had mature fibrotic cancer stroma whereas $32.9 \%$ had intermediate stroma and $14.5 \%$ had immature stroma. The type of stromal reaction was not influenced by the location of the tumour. Five year and 10 year survival rates were worst in the group with immature stroma, better in the intermediate stroma group, and best in the mature stroma group (fig 4). Cancer specific five year survival rates for each group were $79.5 \%, 54.5 \%$, and $27.2 \%$, respectively (table 1 ).

\section{Correlation between maturation of fibrotic cancer stroma and tumour budding}

There was a statistically significant correlation between the category of fibrotic stroma and the degree of tumour budding (table 2). Higher scores of tumour budding were more frequent in immature stroma. Because both variables had a statistically significant impact on survival by multivariate analysis (table 3), maturation of fibrotic cancer stroma and tumour budding influence patient survival independently.

Table 1 Survival values by maturation of fibrotic cancer stroma

\begin{tabular}{|c|c|c|c|c|c|c|c|}
\hline \multirow{2}{*}{$\begin{array}{l}\text { Maturation of } \\
\text { fibrotic cancer } \\
\text { stroma }\end{array}$} & \multirow[b]{2}{*}{ No of patients } & \multicolumn{4}{|c|}{ Prognosis } & \multicolumn{2}{|c|}{ Survival rate $(\%)$} \\
\hline & & $\begin{array}{l}\text { Cancer } \\
\text { death }\end{array}$ & Alive & $\begin{array}{l}\text { Non-cancer } \\
\text { deaths }\end{array}$ & $\begin{array}{l}\text { Dead: cause } \\
\text { unknown }\end{array}$ & $5 y$ & $10 y$ \\
\hline Mature & $453(52.6 \%)$ & 103 & 189 & 123 & 38 & 79.5 & 74.5 \\
\hline Intermediate & $284(32.9 \%)$ & 143 & 80 & 42 & 19 & 54.5 & 45.2 \\
\hline Immature & $125(14.5 \%)$ & 92 & 14 & 16 & 3 & 27.2 & 20.8 \\
\hline Total & $862(100 \%)$ & 338 & 283 & 181 & 60 & & \\
\hline
\end{tabular}

In the data set of 862 patients, long term survival rates decreased in proportion to maturation of fibrotic stroma. 
Table 2 Correlation between maturation of fibrotic cancer stroma and degree of tumour budding

\begin{tabular}{llllll}
\hline \multirow{2}{*}{$\begin{array}{l}\text { Maturation of fibrotic } \\
\text { cancer stroma }\end{array}$} & \multicolumn{2}{l}{ Score of tumour budding } & \\
\cline { 2 - 5 } & $\mathbf{I}$ & II & III & IV & Total \\
\hline Mature & $300(66.2 \%)$ & $82(18.1 \%)$ & $48(10.6 \%)$ & $23(5.1 \%)$ & $453(100 \%)$ \\
Intermediate & $72(25.4 \%)$ & $59(20.8 \%)$ & $81(28.5 \%)$ & $72(25.4 \%)$ & $284(100 \%)$ \\
Immature & $8(6.4 \%)$ & $17(13.6 \%)$ & $36(28.8 \%)$ & $64(51.2 \%)$ & $125(100 \%)$ \\
Total & 380 & 158 & 165 & 159 & 862 \\
\hline
\end{tabular}

Values in the table represent case numbers.

$\rho=0.62 ; z=18.08 ; p<0.0001$ (Spearman's test).

Table 3 Multivariate analysis on maturation of fibrotic cancer stroma and other prognostic factors. Maturation of fibrotic cancer stroma was significant by multivariate analysis

\begin{tabular}{llllll}
\hline Variables selected* & Coefficient & SE & $\chi^{2}$ & HR (95\% CI) & p Value \\
\hline Nodal involvement & 0.51 & 0.08 & 43.01 & $1.67(1.43-1.94)$ & $<0.0001$ \\
Intensity of tumour budding & 0.62 & 0.13 & 21.64 & $1.85(1.43-2.40)$ & $<0.0001$ \\
Maturation of fibrotic cancer stroma & 0.35 & 0.09 & 16.38 & $1.41(1.20-1.67)$ & $<0.0001$ \\
Tumour phenotype & 0.34 & 0.10 & 11.46 & $1.41(1.15-1.71)$ & 0.0007 \\
Extramural venous invasion & 0.32 & 0.11 & 7.83 & $1.38(1.10-1.72)$ & 0.0051 \\
\hline
\end{tabular}

*Only variables found to be significant in the model using a stepwise Cox regression model were included. The variables analysed and their categories and scores were: (1) age $(<60=0, \geqslant 60=1)$; (2) maximum diameter of tumour $(<5 \mathrm{~cm}=0, \geqslant 5 \mathrm{~cm}=1)$; (3) tumour phenotype (well=0, moderate = 1, poor/mucinous = 2); (4) nodal involvement ( $\mathrm{pNO}=0, \mathrm{pN} 1=1, \mathrm{pN} 2=2)$; (5) extramural venous invasion (absent =0, present = 1); $(6)$ intensity of tumour budding (score $\mathrm{I} / \mathrm{II}=0$, score $\mathrm{III} / \mathrm{IV}=1$ ); (7) maturation of fibrotic cancer stroma (mature $=0$,

intermediate $=1$, immature $=2$ ).

$\mathrm{HR}$, hazard ratio; $\mathrm{Cl}$, confidence interval.

\section{Correlation between maturation of fibrotic cancer stroma and the distribution of T cells and myofibroblasts}

The average number of $\mathrm{T}$ cells was $404 / 500 \mu \mathrm{m}$ diameter field in the region of mature fibrotic stroma, compared with 221 and 107 in the regions of intermediate and immature fibrotic stroma, respectively (fig 5 ).

Pervasive distribution of myofibroblasts was observed in $25 \%$ of tumours with mature fibrotic stroma compared with $47 \%$ in intermediate fibrotic stroma (table 4 ). On the other hand, all tumours with immature fibrotic stroma demonstrated pervasive distribution of myofibroblasts.

\section{DISCUSSION}

Accumulation of knowledge regarding the extracellular matrix suggests that an understanding of the mechanisms underlying tumour cell invasion requires analysis of the complex interactions between neoplastic cells and the surrounding matrix. ${ }^{13}$ It has been argued that the process of dedifferentiation and dissociation of neoplastic cells at the invasive edge is the first and essential step in tumour invasion. ${ }^{14}$ Based on the observation that this process is always strictly confined to the tumour invasive front, inductive signals from the host microenvironment were thought to be involved in initiating and maintaining this rapid and even reversible phenotypic shift through activating or repressing the preformed genetic programme of tumour cells. ${ }^{14}$ Conversely, collagen I protein in the centre of the tumour, produced in the desmoplastic response, was reported to be a microenvironmental factor which inhibits the dedifferentiation process of the tumour. ${ }^{15}$ The concept of maturation of fibrotic cancer stroma which we propose would be compatible with this scenario; it has been shown to have a significant correlation with the intensity of tumour budding - that is, histological dedifferentiation, including dissociation of cancer cells and the first step of invasion, ${ }^{16}$ which influences survival outcome in colorectal cancer patients. ${ }^{11} 1617$ Intermediate fibrotic stroma and immature fibrotic stroma were practically confined to the tumour invasive front in particular tumours; and the tumour centre was composed of fine and mature fibres stratified into multilayers (the mature stroma). Based on these findings, intermediate and immature fibrotic stroma seem to be transitory phenotypes which facilitate dedifferentiation of cancer cells whereas mature stroma is a later and more stable phenotype which may reduce the invasive activity of neoplastic cells whether they are located in the tumour centre or invasive front.

Univariate and multivariate analyses showed that the categorised fibrotic cancer stroma significantly correlated with patient survival outcome. Some modulators essential in

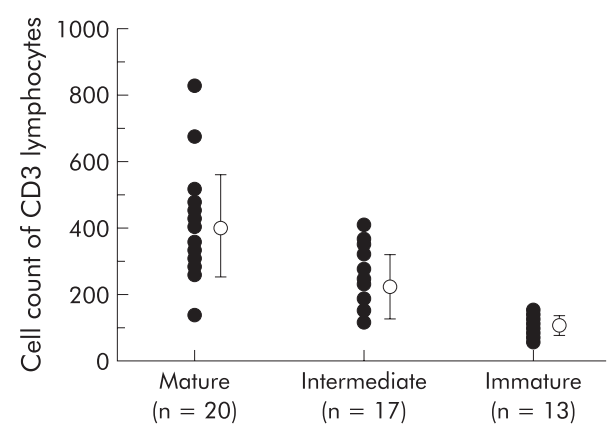

Figure 5 Correlation between maturation of fibrotic cancer stroma and number of CD3 positive lymphocytes. Note that the number of CD3 lymphocytes decreases according to loss of maturation of the surrounding stroma. Mature versus intermediate, $p=0.0002$; intermediate $v$ immature, $p=0.0003$; mature $v$ immature, $p<0.0001$ (unpaired $t$ test). Bar indicates SD. 
Table 4 Correlation between maturation of fibrotic cancer stroma and distribution of myofibroblasts

\begin{tabular}{llcl}
\hline \multirow{2}{*}{$\begin{array}{l}\text { Maturation of fibrotic } \\
\text { cancer stroma }\end{array}$} & \multicolumn{2}{l}{ Localisation pattern of myofibroblast } & \\
\cline { 2 - 4 } & Confined & Pervasive & Total \\
\hline Mature & $15(75.0 \%)$ & $5(25.0 \%)$ & $20(100 \%)$ \\
Intermediate & $9(52.9 \%)$ & $8(47.1 \%)$ & $17(100 \%)$ \\
Immature & $0(0 \%)$ & $13(100 \%)$ & $13(100 \%)$ \\
Total & $24(48.0 \%)$ & $26(52.0 \%)$ & \\
\hline Values in the table represent case numbers. & & \\
$U=118.50 ; z=-3.76 ; p<0.0001$ (Mann-Whitney $U$ test) & & \\
\hline
\end{tabular}

tumour differentiation, cell migration, and tumour progression derive from fibroblasts. ${ }^{18-22}$ Taking into account the difference in survival outcome and degree of tumour budding between mature and intermediate fibrotic stroma, synthesis of "keloid-like" collagen, which stained excessively with anti-type 1 collagen antibody, ${ }^{10}$ might be presumed to represent the deviant function of fibroblasts. It would consequently seem that tumour behaviour and clinical outcome can be mediated by the nature of the tumour stroma.

Shürlch et al classified stromal reactions into three types (precocious, synchronous, and late) in breast cancer in terms of the relationship between the distribution of myofibroblasts and location of the cancer front. ${ }^{23}$ Being different from breast cancer, myofibroblasts were observed to precede carcinoma cells into the reactive fibrous zone ${ }^{24}$ in rectal cancers (that is, precocious type by Shürlch's classification). The pattern of localisation of myofibroblasts in the reactive fibrous zone surrounding the invasive margin of the cancer was related to the categories of fibrotic cancer stroma. Myofibroblasts were distributed pervasively in the immature fibrotic stroma whereas they were confined to just around the invasive front in mature stroma and intermediate stroma in $75 \%$ and $53 \%$ of cases examined, respectively. The myofibroblast has recently been spotlighted because of its production of lytic enzymes able to degrade the basement membrane surrounding tumour glands, and its synthesis of the extracellular matrix components in the tumour stroma, which could subsequently alter the adhesive and migratory properties of epithelial cancer cells. $^{25}$

Although the decrease in lymphocyte infiltration in colorectal primary tumours has long been believed to correlate with poor prognosis, ${ }^{26-28}$ the mechanism by which tumours inhibit lymphocyte locomotion was not clear. Lieubeau et al reported that myofibroblasts may prevent penetration of immune cells within tumours, creating a physical barrier against an immune reaction while promoting tumour growth and progression, due to their contractile properties and their associated extracellular matrix. ${ }^{29}$ The results of in vitro experiments would also support our findings-namely, $\mathrm{T}$ lymphocytes infiltrated less in immature fibrotic stroma, where myofibroblasts were extensively distributed, than in mature and intermediate fibrotic stroma. The above results might suggest that immature fibrotic cancer stroma may serve cancer as a "cocoon", ${ }^{30}$ hindering immune cells from reaching tumours and facilitating the survival of neoplastic cells. This could be an important reason for the poor prognosis associated with immature stroma, which was verified in our cohort of 862 cases.

To summarise, classification of fibrotic cancer stroma in advanced rectal adenocarcinoma was made histologically, and this could help our understanding of the role of the fibrous stromal response in modulating tumour behaviour and host immune reactions. We suggest that fibroblasts and myofibroblasts, which are thought to represent two isoformic transitions of a common ancestor cell that develop in response to a functional demand or a change within the microenvironment, ${ }^{23}$ determine the nature of the stroma. By gaining a clear knowledge about the mechanisms which establish intermediate and immature fibrotic stroma, it may be possible to find new therapeutic strategies for limiting tumour aggressiveness.

\section{Authors' affiliations}

H Ueno, Department of Surgery I, National Defence Medical College, Japan

A M Jones, Molecular and Population Genetic Department, Cancer Research, UK

K H Wilkinson, Department of Research Records, St Mark's Hospital, UK J R Jass, Department of Pathology, McGill University, Canada I C Talbot, Academic Department of Pathology, St Marks Hospital, UK

\section{REFERENCES}

1 Liotta LA, Rao CN, Barsky SH. Tumor invasion and the extracellular matrix. Lab Invest 1983;49:636-49.

2 Barsky SH, Gopalakrishna R. Increased invasion and spontaneous metastasis of BL6 melanoma with inhibition of the desmoplastic response in C57 BL/6 Mice. Cancer Res 1987;47:1663-7.

3 Nakanishi H, Oguri K, Takenaga K, et al. Differential fibrotic stromal response of host tissue to low- and high-metastatic cloned lewis lung carcinoma cells. Lab Invest 1994;70:324-32.

4 Halvorsen TB, Seim EVA. Association between invasiveness, inflammatory reaction, desmoplasia and survival in colorectal cancer. J Clin Pathol 1989:42:162-6.

5 Schürch W, Lagacé R, Seemayer TA. Myofibroblastic stromal reaction in retracted scirrhous carcinoma of the breast. Surg Gynecol Obstet 1982;154:351-8.

6 Shimosato Y, Hashimoto T, Kodama T, et al. Prognostic implications of fibrotic focus (scar) in small peripheral lung cancer. Am J Surg Pathol 1980;4:365-73.

7 Kaiiyama K, Maeda T, Takenaka K, et al. The significance of stromal desmoplasia in intrahepatic cholangiocarcinoma. A special reference of 'scirrhous-type' and 'nonscirrhous-type' growth. Am J Surg Pathol 1999;23:892-902.

8 Breuninger H, Schaumburg-Lever G, Holzshuh J, et al. Desmoplastic squamous cell carcinoma of skin and vermilion surface. A highly malignant subtype of skin cancer. Cancer 1996;79:915-19.

9 Dvorak HF. Tumors: wounds that do not heal; similarities between tumor stroma genaration and wound healing. $N$ Engl J Med 1986;315:1650-9.

10 Ueno $H$, Jones A, Jass JR, et al. Clinicopathological significance of the 'keloidlike' collagen and myxoid stroma in advanced rectal cancer. Histopathology 2002;40:327-34.

11 Ueno H, Murphy J, Jass JR, et al. Tumour 'budding' as an index to estimate the potential of aggressiveness in rectal cancer. Histopathology 2002;40: 127-32.

12 Ueno $\mathrm{H}$, Mochizuki $\mathrm{H}$, Shinto $\mathrm{E}$, et al. Histological indices in biopsy specimens for estimating the probability of extended local spread in rectal cancer. Cancer 2002;94:2882-91.

13 lozzo RV. Editorial: Tumor stroma as a regulator of neoplastic behavior. Lab Invest 1995;73:157-60.

14 Gabbert H, Wagner R, Moll R, et al. Tumour dedifferenatiation: an important step in tumour invasion. Clin Exp Metast 1985;3:257-79.

15 Hewitt RE, Powe DG, Carter GI, et al. Desmoplasia and its relevance to colorectal tumour invasion. Int J Cancer 1993;53:62-9.

16 Hase K, Shatney C, Johnson D, et al. Prognostic value of tumor "budding" in patients with colorectal cancer. Dis Colon Rectum 1993;36:627-35.

17 Ueno H, Mochizuki H, Hatsuse K, et al. Indicators for treatment strategies of colorectal liver metastases. Ann Surg 2000;231:59-66. 
18 Speirs V Ray KP, Freshney RI. Paracrine control of differentiation in the alveolar carcinoma, A549, by human faetal lung fibroblasts. Br J Cancer $1991 ; 64: 693-9$

19 Basset $\mathrm{P}$, Bellocq JP, Wolf $\mathrm{C}$, et al. A novel metalloproteinse gene specifically expressed in stromal cells of breast carcinoma. Nature 1990 348:699-704.

20 Gleave M, Hsieh JT, Gao C, et al. Acceleration of human prostate cancer growth in vivo by factors produced by prostate and bone fibroblasts. Cancer Res 1991;51:3753-61.

21 Lieubeau B, Garrigue L, Barbieux I, et al. The role of transforming growth factor beta 1 in the fibroblastic reaction associated with rat colorectal tumor development. Cancer Res 1994;54:6526-32.

22 Stoker M, Gherardi E, Perryman M, et al. Scatter factor is a fibroblast-derived modulator of epithelial cell mobility. Nature 1987;327:239-42.

23 Shürlch W, Seemazer TA, Gabbiani G. Myofibroblast. In: Sternberg S, ed. Histology for pathologists, 2nd edn. Philadelphia: Lippincott-Raven Publishers, 1997:129-65.

24 Ohtani H, Nagai T, Nagura H. Similarities of in situ mRNA expression between gelatinase A (MMP-2) and type I procollagen in human gastrointestinal carcinoma: comparison with granulation tissue reaction. Jpn J Cancer Res 1995;86:833-9.
25 Martin M, Pujuguet P, Martin F. Role of stromal myofibroblasts infiltrating colon cancer in tumor invasion. Pathol Res Pract 1996;192:712-17

26 Black MM, Speer FD, Opler SR. Structural representations of tumor-host relations in mammary carcinoma. Biologic and prognostic significance. Am J Clin Pathol 1956;26:250-65.

27 Jass JR, Atkin WS, Cuzick J, et al. The grading of rectal cancer: histological perspectives and a multivariate analysis of 447 cases. Histopathology 1986; 10:437-59

28 Ueno H, Mochizuki H, Hase K, et al. A study on the clinical significance of lymphatic tissue responses against rectal cancer. J Jpn Soc Coloproctol 1994:47:430-41.

29 Lieubeau B, Heymann MF, Henry F, et al. Immunomodulatory effects of tumorassociated fibroblasts in colorectal-tumor development. Int $J$ Cancer 1999;81:629-36

30 Dvorak HF, Dvorak AM, Manseau EJ, et al. Fibrin gel investment associated with line 1 and line 10 solid tumor growth, angiogenesis and fibroplasia in guinea pigs. Role of cellular immunity, myofibroblasts, microvascular damage and infarction in line 1 tumor regression. J Nat Cancer Inst 1979;62:1459-73. 